\title{
Si possono trarre solo le conclusioni o anche le conseguenze?
}

\section{Giuseppe Patota}

PUBBLICATO: 15 GENNAIO 2019

Quesito:

Diversi utenti ci hanno chiesto se sia corretto dire o scrivere trarre le conseguenze e non, piuttosto, soltanto le conclusioni.

Si possono trarre solo le conclusioni o anche le conseguenze?

Siamo obbligati a rispondere che la correttezza della formula trarre le conseguenze è garantita non soltanto dall'uso frequente che se ne fa (e non da oggi: una rapida ricerca in Google libri ci ha consentito di individuarne varie occorrenze in testi risalenti all'Ottocento), ma anche dal fatto che ben tre eccellenti vocabolari dell'italiano contemporaneo la considerano addirittura esemplare, al punto di usarla per la fraseologia che illustra uno dei possibili significati del verbo, quello di 'dedurre'. Nella voce che il Grande dizionario italiano dell'uso diretto da Tullio De Mauro (Torino, UTET, 1999) dedica a trarre si legge: "Che conseguenza vorresti trarre?"; nel Treccani (Roma, Istituto della Enciclopedia Italiana, 2015) troviamo: "a voi trarne le conseguenze, o la morale"; infine, nel diffusissimo Zingarelli 2019 (Bologna, Zanichelli, 2018) abbiamo: "traete voi le conseguenze". Il quadro può essere ulteriormente arricchito dal "trarre le conseguenze" che si legge a corredo della definizione della parola conseguenza nei Sinonimi e contrari della Treccani (Roma, Istituto della Enciclopedia Italiana, 2003). A questo punto è d'obbligo - e il bisticcio ci sarà perdonato - trarre la conclusione che si possono trarre anche le conseguenze.

\section{Cita come:}

Giuseppe Patota, Si possono trarre solo le conclusioni o anche le conseguenze?, "Italiano digitale", 2019, VIII, 2019/1 (gennaio-marzo) , pp. 5.

DOI: $10.35948 / 2532-9006 / 2019.3053$

\section{Copyright 2019 Accademia della Crusca}

Pubblicato con licenza creative commons CC BY-NC-ND 